\title{
PLINIO IL VECCHIO E LA SUA STORIA DELL'ARTE ANTICA
}

Parole chiave: Plinio il Vecchio, Storia naturale, Duride di Samo, Senocrate di Atene, Antigono di Caristo, Apollodoro di Atene, Pasitele, Marco Terenzio Varrone

\section{Scritti antichi sull'arte}

Le opere antiche che trattavano d'arte sono quasi completamente scomparse. E' una grave perdita poiché nel mondo Greco la riflessione su questo tema iniziò molto presto e con il tempo essa si sviluppò in quantità e in qualità. Dopo i Greci, anche i Romani apportarono un loro importante contributo a questo argomento. Dalle fonti sappiamo che la letteratura sull'arte era suddivisa in vari generi: storiografia artistica, trattati sui singoli generi artistici e sulle varie tecniche, biografie di artisti, monografie sui singoli monumenti, guide, ecc.

Di tutta questa vasta produzione nulla ci è arrivato degli scritti greci, mentre ci sono pervenute solamente tre opere dell'età romana: il trattato Sullarchitettura, scritto da Vitruvio, lopera enciclopedica di Plinio il Vecchio dal titolo Storia naturale, che nei singoli capitoli include passi più o meno lunghi su diverse tecniche artistiche, ed infine la Periegesi della Grecia di Pausania, volume dedicato ai viaggiatori romani di cultura più elevatare scritto in lingua greca.

I capolavori antichi, soprattutto gli originali greci, sono giunti fino ai nostri giorni in numero molto limitato. Le opere artistiche greche ci sono note soprattutto attraverso copie romane, siano esse statue o, più raramente, dipinti. Questo rende ancora più importanti, oltre ai documenti epigrafici e papirologici, gli scritti degli autori antichi, che nella maggior parte dei casi videro personalmente gli originali. I testi di Vitruvio, Plinio e Pausania 
sono di valore eccezionale non soltanto in quanto unici testi della ricca letteratura storico-artistica conservati in toto, ma anche poiché ci forniscono una moltitudine di informazioni che ci restituiscono un'immagine più completa e verosimile delle arti antiche.

Accanto ai contributi fondamentali di questi tre autori sono da considerare anche, tra le fonti storico-artistiche antiche, tutti gli scritti del genere letterario della ekphrasis, ossia quelle descrizioni retoriche di opere artistiche (sia reali sia immaginarie) che a volte costituiscono dei testi a sé stanti e a volte si trovano incluse, come digressioni, in libri di diverso argomento. ${ }^{1}$

In un terzo gruppo vanno annoverati infine i numerosi frammenti di vari scrittori antichi che, pur trattando tematiche diverse da quelle artistiche, accennano a vari aspetti dell'arte antica. Gli archeologi e i filologi moderni hanno raccolto tutti questi passaggi in un corpus molto voluminoso, che contribuisce in modo importante alla nostra conoscenza di questo argomento.

Quelle elencate sono tutte le fonti delle quali possiamo disporre oggi. Plinio il Vecchio invece, quando compilava i suoi libri storico-artistici verso la seconda metà del I secolo dopo Cristo, disponeva di unampia biblioteca, oggi scomparsa, che gli offriva una vasta panoramica sulla problematica storico-artistica. La maggior parte di queste opere e di questi autori (index autorum), sia romani (ex auctoribus) che greci (externis), sono elencati dallo stesso Plinio nel primo libro della sua Storia naturale, ed ulteriori riferimenti e citazioni si ritrovano sparsi ovunque nel testo. ${ }^{2}$ Da queste sue note, ed anche dai frammenti simili di altri scrittori che si sono conservati, è possibile rendersi conto della quantità immensa di letteratura storico-artistica, scritta soprattutto in lingua greca, che è andata perduta per sempre.

1 Famose ekphraseis sono la descrizione dello scudo di Achille nell'Iliade di Omero (XVIII, 468-617) e il pendant nell'Eneide di Virgilio (VIII, 608-731, lo scudo di Enea).

2 Plinio il Vecchio, nel suo primo libro della Storia naturale, nomina in modo esplicito come fonti per la storia dell'arte greca i seguenti autori (qui sono elencati per ordine alfabetico; cf. Appendix): Antigono (I, 33; I, 34), Apelle (I, 35), Asclepiodoro (I, 35), Duride (I, 34), Eufranore (I, 35), Eliodoro (I, 34; I, 35), Senocrate (I, 34), Melanzio (I, 35), Menecmo (I, 33; 1, 34), Metrodoro (I, 35), Pasitele (I, 33; I, 34). 


\section{Le fonti greche e romane di Plinio il Vecchio}

I primi trattati storico-artistici furono redatti nel VI e V secolo a.C. ad opera dagli artisti stessi. Fra questi figurano l'architetto Teodoro di Samo, lo scultore Policleto di Argo ed il pittore Agatarco di Samo. Si trattava di scritti di natura tecnica inerenti soprattutto le problematiche delle proporzioni e della prospettiva, analizzate su basi matematico-geometriche.

La trattattistica si sviluppò soprattutto nellambito delle scuole pittoriche del IV secolo a.C. Tra i rappresentanti della cosiddetta scuola attica si distinse più degli altri, per la sua versatilità, Eufranore di Corinto, celebrato sia come scultore in marmo sia come pittore, che scrisse il trattato Sulla simmetria e sui colori. La cosiddetta scuola di Sicione e soprattutto il suo principale maestro, il pittore Panfilo di Anfipoli, ebbero poi un ruolo decisivo per l'inclusione della pittura fra le artes liberales. Tra i rappresentanti di questa scuola si ricordano Melanzio di Sicione e Apelle di Colofone che composero ciascuno una propria opera intitolata Sulla pittura. Plinio cita ancora, tra gli autori di trattati sulla pittura del IV secolo a.C., Asclepiodoro di Atene che era sia pittore sia scultore in bronzo.

La letteratura storico-artistica, quella che almeno in parte combacia con il concetto moderno di storia dell'arte, ebbe suo inizio nel primo ellenismo. Tre sono i nomi che ci sono noti e che Plinio menziona: Duride di Samo, Senocrate di Atene ed Antigono di Caristo; è tuttavia verosimile che la produzione letteraria di questo genere dovesse essere molto ricca e varia.

Cronologicamente la fonte più antica dalla quale attinse Plinio, è Duride di Samo che nacque in Sicilia, ad Eraclea Minoa, nel 340 a.C. La sua famiglia, che tra i propri antenati annoverava anche Alcibiade, era originaria di Samo, ma fu espulsa dall'isola per volontà di Filippo II di Macedonia. Duride si formò ad Atene, dove studiò presso il filosofo Teofrasto, e fu autore di numerose opere.

Dopo l’amnistia proclamata per tutti gli esuli di Samo nel 332 a.C. dal generale macedone Perdicca, Duride ritornò sull'isola paterna. Intorno al 300 a.C. ne divenne il tiranno e la governò fino alla morte, avvenuta nel 270 a.C. circa.

Di tutti i suoi scritti, quelli più apprezzati furono quelli di carattere storico. Fra questi ultimi si ricordano gli Annali dei Samii, di cui restano 5 
frammenti, aventi per oggetto la storia locale di Samo, la Storia di Agatocle, una monografia in 4 libri sul tiranno di Siracusa della quale ci sono pervenuti 6 frammenti, e la Storia greca, che sarebbe più corretto chiamare Storia macedone, composta da 23 libri che descrivevano la storia della Macedonia dalla morte di Aminta, padre di Filippo II, nel 370 a.C., al 281 a.C., anno in cui morì il generale Lisimaco, che per breve tempo regnò sul trono macedone (284-281 a.C.).

Duride compose, oltre agli studi storici, anche altre opere di varia erudizione, secondo il modello della scuola peripatetica. Sono inclusi in questa produzione i trattati sulla teatrologia intitolati Sulla tragedia, Su Sofocle ed Euripide e Sugli Agoni (quest'ultimo riguarda in modo specifico lo svolgimento delle rappresentazioni teatrali). Ai problemi interpretativi dedicò lo scritto Problemi omerici, ispirata all'omonima opera di Aristotele, e ad unaltra raccolta dello stesso filosofo, quella delle Costituzioni delle città greche, Duride si ispirò per scrivere quello intitolato Sulle leggi.

Nel nostro contesto, di tutto il vasto opus dellautore, interessano specificatamente due trattati d'arte: Sulla pittura e Sui rilievi. Gli storiografi della letteratura antica li elencano fra i suoi lavori di carattere storico, poiché essi furono scritti con i mezzi espressivi della storiografia. La storiografia di Duride rappresenta un passaggio fondamentale verso un tipo di storia drammatizzata qual è quella del primo ellenismo.

Duride costruisce la narrazione storica basandosi su elementi drammatici e quasi teatrali, probabilmente sulla falsa riga dellopera di Teofrasto Sulla storiografia, oggi purtroppo perduta. Al lettore i personaggi vengono presentati in modo così vivace da farli sembrare degli attori posti sulla scena teatrale; di loro lo scrittore descrive in tutte le sfaccettature, delineandone efficacemente sia le caratteristiche fisiche sia, soprattutto, quelle psicologiche.

Duride impernia la propria narrazione sui protagonisti dell'evento storico descritto e si spinge al punto da sfiorare il genere biografico. Con questi stessi principi vennero redatti anche i due trattati d'arte, Sulla pittura e Sui rilievi. Entrambi furono concepiti come una serie di biografie di artisti, per ciascuno dei quali l'autore evidenzia il punto di massima espressione artistica, o apice (gr. akme, lat. floruit), che si concreta naturalmente con la realizzazione del loro massimo capolavoro. 
Plinio il Vecchio cita Duride in un passo del libro XXXIV, e dall'autore greco prende l'affermazione che Lisippo non seguì nessun maestro, ma prese piuttosto come modello la natura stessa (XXXIV, 61): Lysippum Sicyonium Duris negat ullius fuisse discipulum, sed primo aerarium fabrum audiendi rationem cepisse pictoris Eupompi responso. eum enim interrogatum, quem sequeretur antecedentium, dixisse monstrata hominum multitudine, naturam ipsam imitandam esse, non artificem.

In questo passo intravediamo le caratteristiche biografiche che dovevano essere tipiche degli scritti sull'arte composti da Duride. Da questi indizi possiamo dedurre che Plinio attingesse da questi soprattutto per gli aneddoti con i quali rese più vivaci i propri passaggi sulla vita e sulle opere degli artisti più famosi.

Senocrate di Atene visse nella prima metà del III secolo a.C. (280-250 a.C. circa). L'apice della sua produzione può essere collocato nel periodo della $130^{\text {a }}$ Olimpiade, ossia tra gli anni 260-257 a.C. Ad Atene frequentò la scuola peripatetica, mentre a Sicione acquisì padronanza nella tecnica scultorea. Plinio il Vecchio lo annovera tra i rappresentanti della scuola di Lisippo, che ebbe il suo akme tra gli anni 296-293 a.C. e che, stando a Pausania e alle testimonianze epigrafiche, proseguì la propria attività fino alla fine del III secolo a.C. Secondo Plinio a questa scuola appartennero anche i tre figli di Lisippo, Laippo, Boeda ed Euticrate, e poi Tisicrate di Sicione, allievo di Euticrate, Charete di Lindo, Fani ed infine Senocrate di Atene, che fu allievo sia di Euticrate sia di Tisicrate. ${ }^{3}$ Senocrate superò entrambi i suoi due maestri per numero di sculture realizzate, ${ }^{4}$ e la sua firma si è conservata sulle tre basi di statue dell'inizio del III secolo a.C. Da queste iscrizioni possiamo dedurre che l'artista intorno all'anno 280 a.C. si fermò per un certo periodo ad Oropo, e poi a Pergamo, dove realizzò il ritratto ufficiale di Filetero. Quest'ultimo è stato riconosciuto dallo studioso moderno P. Moreno nella copia romana in Villa dei Papiri ad Ercolano (Moreno 1994, 259).

3 Plinio il Vecchio, Storia naturale, XXXIV, 66-67 (Laippus, Boëdas, Euthycrates, Tisicrates); XXXIV, 41 (Chares); XXXIV, 80 (Phanis); XXXIV, 83: Xenocrates, Tisicratis discipulus, ut alii, Euthycratis, vicit utrosque copia signorum. et de sua arte conposuit volumina.

Supra n. 3. 
Sulla base quanto riporta Plinio, Senocrate di Atene fu autore di due trattati intitolati De toreutice e De pictura. ${ }^{5}$ Numerosi studiosi, che in epoca moderna approfondirono la questione delle fonti greche e latine di Plinio, concordano nel ritenere che sia proprio Senocrate, fra tutti gli altri autori menzionati, la sua fonte principale per la storia dellarte antica.

Secondo il parere di B. Schweitzer, che per primo nel 1932 ne ricostruì lopera, spetta proprio a Senocrate di Atene il ruolo di padre della storia dell'arte. Egli infatti fu il primo ad applicare l'idea del progresso evoluzionistico del filosofo Democrito alla storia delle arti visive. Va sottolineato che fu proprio in quel periodo, grazie alla scuola di Sicione, che la pittura trovò finalmente il suo posto tra le arti liberali. Fu in seguito a questo fatto che la pittura, in particolare quella su tavola, divenne unarte basilare nell'educazione dei liberi cittadini e ad essa venne riconosciuto un valore pari a quello della poesia.

Senocrate di Atene, nei suoi scritti, concepì la storia dellarte come una successione di innovazioni costituenti un processo evolutivo continuo. In questo quadro interpretativo egli assegnò ai grandi maestri del passato il ruolo fondamentale di scopritori (protoi heuretai). Larte seguì quindi uno sviluppo costante fino ad approdare al naturalismo ed alla perfezione che, secondo il parere di Senocrate, si manifestò tra gli scultori nella persona di Lisippo e tra i pittori in quella di Apelle.

In questa progressione ogni artista migliorò il suo predecessore: ad ogni heuretes, "scopritore" seguì il suo teleiotes, "perfezionatore". Ognuno di essi fu limitato dal difetto di una o di unaltra qualità che il suo successore possedette in misura maggiore. Nella bronzistica l'idea di una serrata, inesorabile consequenzialità dellarte greca, si traduce nella sequenza Fidia - Policleto Mirone - Pitagora. Mirone è numerosior, "più ritmico", di Policleto, mentre Pitagora sopravanza lo stesso Mirone per la cura del dettaglio; Lisippo, non a caso senza maestri, superò tutti.

Il paradigma è perfettamente omologo anche in pittura. La sequenza Cimone - Polignoto - Apollodoro - Zeusi - Parrasio, tutti appartenenti alle

5 Plinio il Vecchio, Storia naturale, XXXIV, 83 (supra n. 3) e XXXV, 68: hanc ei gloriam concessere Antigonus et Xenocrates, qui de pictura scripsere, praedicantes quoque, non solum confitentes. 
grandi scuole del IV secolo a.C., scandisce le tappe che conducono alla piena pittura tonale e prospettica, quella che rispecchia la percezione visiva della realtà, e che avrà il suo maggior esponente in Apelle.

Fu U. von Wilamowitz-Moellendorf, con una monografia del 1881, a scoprire la personalità e l'opera di Antigono di Caristo (Eubea). Questi fu allievo del filosofo Menedemo di Eretria e, in età matura, viaggiò nei territori che circondano il mare Egeo. Durante questi viaggi continuò la propria attività di scultore e raccolse materiale per i propri scritti.

Alla fine del III secolo a.C. si fermò a Pergamo, dove continuò la sua attività di scultore e dove scrisse La vita dei filosofi. Lultima delle biografie comprese in essa si conclude nell'anno 205 a.C., l'anno in cui molto probabilmente morì.

Le sue opere scultoree sono scomparse ed il tentativo di attribuirgli il cosiddetto gruppo di Pasquino è pura speculazione.

Plinio il Vecchio lo cita come autore di due scritti darte: la De toreutice e la De pictura. ${ }^{6}$ Per queste due raccolte, l'autore prese a piene mani dall'opera di Senocrate, arricchendola però con i dati biografici e gli aneddoti di Duride e con ulteriori proprie ricerche biografiche. ${ }^{7}$ Per questa ragione egli può essere considerato come l'antesignano del moderno storico dell'arte.

E' da evidenziare inoltre che Antigono, a differenza di Senocrate, rivalutò Prassitele, noto soprattutto per le opere in marmo, e da lui ritenuto il maggior scultore attico dell'arte tardo classica. Questa valutazione fa di Antigono il precursore del classicismo antico, inteso come forma di ammirazione dell'arte attica del periodo classico e tardo classico, che si irradiò proprio dalla corte di Pergamo.

La grande influenza che Antigono esercitò sulla storiografia dell'arte antica, soprattutto quella romana, è da attribuire a Varrone, che spesso citò i suoi scritti d'arte.

6 Plinio il Vecchio, Storia naturale, XXXIV, 84: Plures artifices fecere Attali et Eumenis adversus Gallos proelia, Isigonus, Pyromachus, Stratonicus, Antigonus, qui volumina condidit de sua arte; XXXV, 68: hanc ei gloriam concessere Antigonus et Xenocrates, qui de pictura scripsere, praedicantes quoque, non solum confitentes.

7 Zenobio (V, 82) ci informa che Antigone trascrisse la firma di Agoracrito, incisa sulla base della statua delle Nemesi di Ramnunte. 
Nel periodo del medio e tardo ellenismo l'idea evoluzionistica della storia dell'arte, nel senso di una consequenzialità delle innovazioni verso la meta del naturalismo artistico, fu concordemente accettata da tutti. I singoli autori si distinsero fra di loro soltanto nella collocazione dell'apice di questo sviluppo artistico, assegnato ad uno o all'altro periodo dell'arte greca. Se da un lato Senocrate di Atene e dopo di lui Antigono di Caristo riconoscono la massima completezza artistica nellopera di Lisippo ed Apelle, entrambi della seconda metà del IV secolo a.C., altri autori a loro successivi sposteranno questo primato indietro nel tempo assegnandolo a Fidia, quale maggior rappresentante dell'Atene dell'età di Pericle.

Lautore principale di questa corrente della storiografia artistica fu senza dubbio Apollodoro di Atene, che nacque nella capitale attica intorno al 180 a.C. Dal 160 a.C. fu allievo di Diogene di Babilonia, filosofo e successore di Crisippo nella direzione della scuola stoica. Da quest'ultimo Apollodoro apprese l'intepretazione allegorica dei miti.

Dopo che Diogene fu inviato a Roma come ambasciatore nel 156 a.C., Apollodoro si spostò ad Alessandria, dove collaborò con il filologo Aristarco di Samotracia. In questo periodo (dal 156 a.C. fino al 145 a.C.) si dedicò soprattutto alla cosiddetta questione omerica ed alla commedia.

Nell'anno 145 a.C. Tolomeo VIII Neo Filopatore pubblicò un eddito con cui espelleva da Alessandria molti intellettuali, per contrasti politici, e tra questi anche Apollodoro che si rifiugiò a Pergamo.

Apollodoro probabilmente morì nella sua città natale intorno a 100 a.C., poiché con questo anno si conclude la narrazione della sua Cronaca.

Egli scrisse numerose opere, tra le quali la più importante si intitola Cronaca, un poema in 4 libri, scritti in trimetri giambici, dei quali ci restano 87 frammenti. Lopera, che fu dedicata al re di Pergamo Attalo II, celebrò gli eventi principali della storia e della letteratura dalla caduta di Troia (datata nel 1184 a.C.) all'anno 110 a.C.

Tra i suoi scritti vanno ricordati anche Sul catalogo delle navi e Sugli dei. Il primo è un commento geografico (in 12 libri) sul famoso Catalogo delle Navi del II canto dell'Iliade; di esso restano 54 frammenti. Nei 66 frammen- 
ti pervenuti del trattato Sugli dei (in 24 libri), Apollodoro cerca di spiegare gli epiteti delle divinità in base alletimologia, con citazioni da vari autori.

Ad Apollodoro è attribuita (in realtà si tratta di uno Pseudo-Apollodoro) anche una delle più complete raccolte mitografiche dell'antichità che ci sia giunta, intitolata Biblioteca. Si tratta di unopera enciclopedica, nella quale lautore tenta di sistematizzare la mitologia greca definendo una genealogia degli dei e riportando ogni singola narrazione mitologica insieme alle sue numerose varianti.

Fra tutte le opere di Apollodoro, la più importante, per quel che riguarda lo sviluppo della storiografia artistica del medio e tardo ellenismo, è senz'altro la Cronaca. In essa l'autore riporta l'elenco degli artisti greci e pone, tra quelli di maggior rilevanza, gli scultori ed i pittori attici del periodo classico e tardo classico.

A differenza di Senocrate, che riconobbe l'apice dello sviluppo artistico nei maestri della scuola di Sicione alla quale apparteneva lui stesso, la visione nostalgica e retrospettiva di Apollodoro si inquadra nella rinascita dell'arte attica intorno al 150 a.C., alla quale fu dato, in tempi più recenti, il termine di classicismo attico oppure neoatticimo. In quel periodo storico gli artisti ed i teorici dell'arte attici rivalutarono la propria tradizione riportando in auge Atene dopo che, per un lungo lasso di tempo, la città era passata in secondo piano rispetto ad altri importanti centri d'arte ellenistica, soprattutto Pergamo, Rodi ed Alessandria. Larte attica così risorse in forma di un nuovo classicismo, e diventò una delle principali tendenze stilistiche del tardo ellenismo. ${ }^{8}$

Il periodo del tardo ellenismo non diede nessun apporto importante alla storiografia artistica. Popolari furono i vari compendia, redatti come una sorta di sistemazione di un sapere già acquisito. Per lo più si trattò di periegesi oppure di elenchi di artisti ordinati secondo il loro livello qualitativo. Fra questi scritti spicca un'opera di Pasitele (108-48 a.C.) in 5 libri,

8 Nella metà del II secolo a.C. visse ad Atene Eliodoro di Atene, che Plinio nominò tra le proprie fonti. Di questo autore sappiamo soltanto che scrisse un'opera in 15 libri sull'Acropoli di Atene, molto probabilmente in forma di periegesi. 
conosciuta con il titolo Mirabilia. ${ }^{9}$ La divisione in 5 libri probabilmente rispecchia i cinque generi dell'arte antica: la toreutica (oppure l'oreficeria), la scultura in bronzo, la pittura, la coroplastica e la scultura in marmo.

A Pasitele, che era originario di Magna Grecia, venne attribuita la cittadinanza romana al tempo della lex Plautia Papiria (89 a.C.). Come centro della sua attività scelse la città di Roma, dove divenne il maggior scultore del suo tempo. ${ }^{10}$ Pasitele padroneggiò quasi tutte le tecniche scultoree, come si deduce dal fatto che vengono ricordate sue opere in marmo, bronzo, argento, avorio e terracotta. ${ }^{11}$ A Roma istituì una propria scuola artistica che non ebbe una diretta relazione con una qualche tendenza stilistica, ma fu piuttosto indirizzata allo studio dalla natura stessa e alla copia dei capolavori precedenti, privilegiando una sorta di eclettismo artistico. Pasitele tese a perfezionare la conoscenza tecnica e la completezza formale, alle quali aggiunse un senso innato per l'eleganza.

Dalle fonti sappiamo che Pasitele realizzò un ritratto cesellato in argento di Roscio bambino, ${ }^{12} \mathrm{e}$ una statua di Giove in avorio per un tempio dedicato da Metello. ${ }^{13}$ Lopera di questo artista fu riconosciuta da M. Borda nel 1953 e, tra i lavori attribuitegli, il più rappresentativo è senza dubbio un kouros di Villa Albani a Roma. Sono poi note alcune sculture di allievi di Pasitele, tra queste soprattutto l'atleta, firmato da un certo Stefano, ${ }^{14}$ ed un gruppo scultoreo di Oreste ed Elettra, ad opera di Menelao, discepolo di Stefano.

9 Plinio il Vecchio, Storia naturale, XXXVI, 39: quinque volumina scripsit nobilium operum in toto orbe; I, 3 e I, 34: Pasitele qui marabilia opera scripsit.

10 Plinio il Vecchio nomina Pasitele in diversi passaggi (XXXIII, 156; XXXV, 156) e gli attribuisce un particolare valore tra gli scultori in marmo (XXXVI, 39-40): ut tradit Varro, admirator et Pasitelis, qui et quinque volumina scripsit nobilium operum in toto orbe. natus hic in Graeca Italiae ora et civitate Romana donatus cum iis oppidis, Iovem fecit eboreum in Metelli aede, qua campus petitur. accidit ei cum in navalibus, ubi ferae Africanae erant, per caveam intuens leonem caelaret, ut ex alia cavea panthera erumperet, non levi periculo diligentissimi artificis. fecisse opera complura dicitur; quae fecerit, nominatim non refertur.

11 Pasitele, a differenza della maggior parte degli altri scultori, realizzò sempre dapprima il bozzetto in terra, che poi traspose in qualche altra tecnica scultorea; cf. Plinio il Vecchio, Storia naturale, XXXV, 156: laudat et Pasitelen, qui plasticen matrem caelaturae et statuariae scalpturaeque dixit et, cum esset in omnibus iis summus, nihil umquam fecit ante quam finxit.

12 Cicerone, De divinatione, 1, 36, 79.

13 Supra n. 10.

14 Plinio il Vecchio, Storia naturale, XXXVI, 33. 
La caratteristica che accomuna i compendi, le periegesi e gli elenchi degli artisti dal II secolo a.C. in avanti, è la concezione classicista della storia dell'arte, che reputa Fidia come il massimo artista di ogni tempo, e che proclama la morte dellarte dopo Lisippo ed Apelle. Partendo da questo presupposto queste raccolte non includono l'arte dell'ellenismo, o al più la valutano secondaria e meno importante. Questa concezione dell'arte greca si riverberò sia sui due autori romani che scrissero sullargomento le opere di maggior importanza, cioé Vitruvio e Plinio, sia su tutti coloro che, e tra questi si ricordano soprattutto Cicerone e Quintiliano, espressero semplici valutazioni su singoli artisti o su vari aspetti dellarte o trattarono di arte soltanto sporadicamente. ${ }^{15}$

Quanto esposto è il quadro completo della letteratura storico-artistica greca così come ce lo presenta Plinio il Vecchio.

Resta da aggiungere che parecchi filologi hanno espresso un certo dubbio sul fatto che Plinio abbia realmente letto tutte le numerose opere che menziona, anche se tutte le sue fonti sono citate con un rigore quasi scientifico. Quindi si ritiene possibile che Plinio abbia preso la maggior parte dei suoi dati dal suo famosissimo predecessore, Marco Terenzio Varrone. ${ }^{16}$

Marco Terenzio Varrone (116-27 a.C.) è il più noto enciclopedista romano e non ha bisogno di presentazioni particolari. Tra tutti gli scritti del suo opus immenso, che comprendeva più di 600 libri, il più significativo per la nostra indagine è la Disciplinarum libri IX. Il testo è purtroppo perduto, ma della sua struttura ci informa S. Agostino, che coltivò l'intenzione di scrivere unopera enciclopedica seguendo rigorosamente lo schema di Varrone. Per questa circostanza conosciamo la successione dei nove libri che costituivano loriginale, e che trattavano le nove discipline delle artes liberales: grammatica, dialettica e retorica in primo grado, geometria, arit-

15 Il concetto della storia dell'arte antica come un progresso culminante nell'arte attica dell'età classica influenzò la successiva valutazione dell'antichità nell'età moderna, a partire da J. J. Winckelmann, e fu la base dei pregudizi negativi sull'arte romana, superati appena con gli scritti di F. Wickoff e A. Riegl.

16 Plinio il Vecchio nomina e cita Varrone in vari passaggi, per esempio XXXIII, 154; XXXIV, 56; XXXV, 11; ecc. 
metica, astronomia, musica, architettura e medicina in secondo. Plinio, per la sua enciclopedia, attinse in gran parte dal libro sullarchitettura, nel quale Varrone trattò, accanto alla tematica principale, anche le altre arti visive, seppur sommariamente.

\section{Plinio il Vecchio come fonte principale per le arti figurative antiche}

Dopo alcuni cenni a toreutica e oreficeria posti in chiusura al libro XXXIII (82-83, 131-132, 139-140, 151, 154-157), il libro XXXIV è quasi integralmente dedicato alla scultura in bronzo (5-92).

Plinio inizia lamentando innanzitutto la decadenza in cui versa la bronzistica del suo tempo (in cui il valore dellopera d'arte è associato unicamente al valore economico del materiale e a null'altro; XXXIV, 5), prosegue affermando che l'origine di questo genere d'arte è corinzia ed infine descrive le principali leghe bronzee antiche: la corinzia, la deliaca e l'eginetica (XXXIV, 6-10).

Plinio riporta che il bronzo può essere fuso in diverse leghe, ognuna delle quali ebbe, nel corso delle varie epoche, utilizzi diversi. Per questo motivo il bronzo è servito dunque per forgiare oggetti disparati. Si sono fatti in bronzo candelabri e simili oggetti decorativi (XXXIV, 11-14) fino ad arrivare alla scultura a tutto tondo, all'inizio raffigurante soltanto gli dei ma più tardi, e sempre più frequentemente, anche figure umane (XXXIV, 15-17). Tra i romani poi queste ultime, dapprima in forma di statue simboliche, si differenziarono via via in tipologie che con il tempo inclusero anche la ritrattistica vera e propria (XXXIV, 18-32). Plinio afferma ancora che la bronzistica è praticata sul suolo italico dai tempi più antichi (XXXIV, 33-34) e a testimonianza di questo fatto menziona la copiosa presenza di sculture in bronzo (XXXIV, 36-37); riporta infine che ai suoi tempi questa forma d'arte si era ormai polarizzata su due estremi opposti: il verismo naturalistico contrapposto ad un gigantismo concretato nei cosiddetti colossi, e tra questi cita quello di Rodi e quello di Nerone (XXXIV, 38-47), da cui avrebbe poi tratto il nome il Colosseo. 
Finalmente, poi, inizia l'elenco cronologico dei bronzisti (XXXIV, 4952 ), al quale segue il prospetto sugli artisti più importanti che si sono cimentati in questo genere (XXXIV, 53-92). Fu Fidia che "aprì per primo le porte dellarte alla scultura in bronzo.". ${ }^{17}$ Dopo di lui si stagliarono per abilità nel V secolo a.C. anche Policleto, Mirone e Pitagora. Il genere della bronzistica conobbe la massima fioritura nell'ultimo quarto del IV secolo a.C., specialmente per opera di Lisippo che sviluppò lo stile del naturalismo. La personalità di questo artista conduce a questo nuovo traguardo, evidente sia nel processo creativo dello scultore, che segue esclusivamente la natura, ${ }^{18}$ sia nel nuovo canone della figura umana, riprodotta ora "come all'occhio appaia essere". ${ }^{19}$

L'excursus sulla pittura che comprende gran parte del libro XXXV (2150) e si apre con note polemiche simili a quelle dell'incipit del XXXIV, sul tema della decadenza della pittura contemporanea, in un mondo dove conta ormai soltanto il valore economico e che per questo apprezza le opere soltanto in quanto realizzate con materiali costosi o preziosi come il marmo o l'oro, anche se del tutto prive di contenuto artistico. Languono quindi le espressioni come la pittura su tavola e parietale, (XXXV, 2-3), e non si salva neppure la ritrattistica romana (XXXV, 4-5) che pur era fiorente nella Roma repubblicana (XXXV, 6-14). A questa introduzione seguono i capitoli nei quali l'autore espone ed approfondisce la questione delle origini della pittura nel mondo greco (le scuole peloponnesiache di Sicione e di Corinto; XXXV, 15-16) e del suo rapido affermarsi in Italia (XXXV, 17-28).

Plinio dedica parecchi capitoli ai materiali impiegati nella tecnica pittorica, ovvero ai pigmenti (XXXI, 30-49), distinguendoli fra colori austeri, cioè secchi e opachi (coprenti, adatti all'affresco) e floridi, lucidi e traspa-

17 Plinio il Vecchio, Storia naturale, XXXIV, 54: primusque artem toreuticen aperuisse atque demonstrasse merito iudicatur.

18 Plinio il Vecchio, Storia naturale, XXXIV, 61: eum enim interrogatum, quem sequeretur antecedentium, dixisse monstrata hominum multitudine, naturam ipsam imitandam esse, non artificem.

19 Plinio il Vecchio, Storia naturale, XXXIV, 65: statuariae arti plurimum traditor contulisse capillum exprimendo, capita minora faciendo quam antiqui, corpora graciliora siccioraque, per quae proceritas signorum maior videretur. non habet Latinum nomen symmetria, quam diligentissime custodiit nova intactaque ratione quadratas veterum statuas permutando, vulgoque dicebat ab illis factos quales essent homines, a se quales viderentur esse. 
renti (per le tavole a encausto). Lelenco dei colori si conclude infine con la considerazione che - similmente a quanto aveva scritto al termine dei capitoli sulla bronzistica -anche la pittura era arrivata a maturare due posizioni contrapposte: il verismo e il gigantismo (XXXV, 51-52).

I capitoli più importanti della storia della pittura antica di Plinio (XXXV, 53-148) sono dedicati alla rassegna degli artisti che sono distinti in tre categorie basandosi su un giudizio di valore: i grandi maestri (XXXV, 54-137), i primis proximi, ossia pittori che per la tecnica raggiunta erano simili ai grandi pittori (XXXV, 138-145) e i non ignobiles, indicando con questo appellativo quelli degni di essere menzionati (XXXV, 146).

I grandi maestri sono divisi secondo le tecniche della pittura antica: tempera (XXXV, 54-121) ed encaustica (XXXV, 122-137). I pittori che usavano la tempera sono ulteriormente distinti in quelli che si dedicarono a soggetti di genere superiore (ritratti, soggetti mitologici) (XXXV, 54-111) ed in specialisti di quelli di tipo inferiore (nature morte, paesaggi). La digressione sulla pittura antica viene conclusa dall'autore con alcuni accenni alle pittrici (XXXV, 147-148) e con brevi annotazioni sulla pittura su tela $(\mathrm{XXXV}, 150) .^{20}$

Nella parte del testo più importante, quella dedicata ai grandi maestri, Plinio descrive brevemente la pittura del V secolo a.C., (XXXV, 57-74) e si intrattiene più ampiamente sull'arte tardo classica del IV secolo a.C. (XXXV, 75-111). I pittori sono divisi tra due scuole, quella scuola tebanoattica, fondata da Eussinida e Aristide il Vecchio, e quella di Sicione, i cui primi rappresentanti furono Eupompo e Panfilo.

Ai grandi maestri del IV secolo a.C., sono dedicati ben 33 capitoli, in cui trovano spazio sia i maestri nella tempera che quelli nellencaustica. Plinio scrive così tanto su questo argomento poiché ritiene che questo periodo dellarte antica rappresenti il culmine della pittura greca. Di questi 33 capitoli, 19 sono dedicati ad Apelle, che secondo lautore "superò tutti in arte".

20 I grandi maestri sono elencati in ordine cronologico, invece primis proximi e non ignobiles per ordine alfabetico; le pittrici infine, seguendo il principio retorico della variatio, secondo ordine alfabetico inverso.

21 Plinio il Vecchio, Storia naturale, XXXV, 79: Verum omnes prius genitos futurosque postea superavit Apelles. 
Il XXXV libro comprende anche alcune nozioni importanti sulla coroplastica (XXXV, 151-165), introdotta in Italia dal Peloponneso nella metà del VII secolo a.C. e che raggiunse un'alta qualità tecnica in Etruria con i lavori dell'artista Vulca di Veio. Lautore conclude il capitolo con la lista dei vari prodotti di questo genere d'arte, (XXXV, 159-165), fra i quali sono da sottolineare soprattutto i sarcofaghi di terracotta.

La digressione sulla scultura in marmo nel libro XXXVI (1-44) segue lo stesso schema delle precedenti sul bronzo e sulla pittura, e quindi si apre con la nota polemica sull'uso dei marmi per le sculture a Roma, apprezzate ormai unicamente in base al valore commerciale del materiale e per nulla invece dal loro valore artistico (XXXVI, 1-8); dopo questa parte introduttiva segue la rassegna degli scultori piu' importanti (XXXVI, 9-44).

A differenza della scultura in bronzo e della pittura, venute alla ribalta soltanto al tempo di Fidia, più esattamente negli anni della $83^{\circ}$ olimpiade (448-445 a.C.), la lavorazione del marmo è più antica ed è contemporanea all'istituzione dei giochi olimpici (nell'anno 776 a.C.). ${ }^{22}$ Agli scultori di epoca arcaica provenienti da Creta e da Chio, seguì Fidia con i suoi due allievi Alcamene e Agoracrito (XXXVI, 15-19).

La maggior parte dei capitoli è dedicata agli importanti scultori del IV secolo a.C., ovvero a Prassitele e al suo figlio Cefisodoto il Vecchio (XXXVI, 20-23), a Scopa e agli altri maestri (Briasside, Timoteo e Leocare), che collaborarono alla decorazione scultorea del Mausoleo di Alicarnasso. La presenza di un capolavoro originale di Timoteo a Roma è poi lo spunto per un elenco delle principali collezioni d'arte romane, che fra l'altro comprendevano anche il famoso gruppo scultoreo di Laocoonte, opera dei tre scultori rodiesi Agesandro, Polidoro e Atenodoro.

Nella seconda parte del libro XXXVI (69-125, 131, 177-179) sono menzionati alcuni dei più importanti monumenti architettonici dell'antichità: obelischi egiziani, soprattutto quelli a Roma, le piramidi egiziane con la famosa Sfinge, il faro di Alessandria, labirinti, il tempio di Artemide ad Efeso, i monumenti a Cizico e infine i capolavori architettonici dell'antica

22 In questo punto Plinio dimentica che nei capitoli dedicati alla pittura (XXXV, 55) lui stesso affermava la vetustità della pittura in quanto poneva le sue origini nell'età dell'Urbe condita (nell'anno 753 a.C.). 
Roma (fra questi il teatro di M. Emilio Scauro, il sistema idraulico con gli acquedotti, i palazzi imperiali, ecc.).

Alla fine del XXXVI libro (184-189) viene presentata, in brevi cenni, la tecnica del mosaico e ricordato il famoso maestro Sosos di Pergamo, mentre nel XXXVII libro (8-10) Plinio accenna ad alcuni nomi di artisti che eccelsero nella tecnica della glittica.

\section{Conclusioni}

Riconsiderando le due principali trattazioni, quelle sulla scultura in bronzo e sulla pittura, si osserva che Plinio il Vecchio adotta uno schema evolutivo: all'iniziale età arcaica segue una prima fioritura in età classica durante il V secolo a.C. e a questa succede l'apice dello sviluppo, con l'arrivo del periodo tardo classico del IV secolo a.C. soprattutto ad opera di Lisippo ed Apelle. Levoluzione della bronzistica per Plinio si conclude nei primi anni del III secolo a.C., ma poi aggiunge una nota in cui afferma che questa ebbe una rinascita successivamente, nel periodo della $156^{\mathrm{a}}$ Olimpiade (fra gli anni 156-153 a.C.). ${ }^{23}$ Similmente conclude anche la rassegna sulla pittura greca, con l'elenco degli artisti che operarono agli inizi del III secolo a.C. A questi ultimi però aggiunge anche alcuni pittori greci scelti tra quelli del medio e tardo ellenismo, con particolare evidenza per i rappresentanti del neoatticismo, Eraclide Macedone e Metrodoro di Atene, entrambi della prima metà del II secolo a.C., e pure Timomaco di Bisanzio che operò a Roma nelletà di Giulio Cesare.

Numerosi studiosi concordano nel ritenere che il concetto che la storia dell'arte greca abbia seguito una parabola evolutiva, sia stato attinto direttamente dalle fonti greche che erano ancora facilmente reperibili nell'età di Plinio. L'autore che ebbe influenza maggiore sull'opera di Plinio, Senocrate di Atene, chiuse la sua rassegna dellarte greca con gli anni 296-293 a.C., ossia con il periodo centrale della scuola di Lisippo e alla quale lui stesso

23 Plinio il Vecchio, Storia naturale, XXXIV, 52: cessavit deinde ars ac rursus olympiade CLVI revisit, cum fuere longe quidem infra praedictos, probati tamen, Antaeus, Callistratus, Polycles, Athenaeus, Callixenus, Pythocles, Pythias, Timocles. 
apparteneva. Apollodoro di Atene e Pasitele invece si collocano nella tradizione neoattica, e quindi nei loro scritti emerge una preferenza per la produzione ateniese del V secolo a.C., con Fidia in testa, e per gli artisti della corrente classicista a loro contemporanei. Il periodo intermedio, compreso tra gli anni 296-293 a.C. e 156-153 a.C., non era coperto dalla letteratura storico-artistica, e da questo fatto i Romani trassero logicamente la conclusione che la produzione artistica di quell'intervallo di tempo non fosse degna di unattenzione particolare.

Nellestetica di Plinio quindi, si intrecciano queste due visioni contrapposte: da una parte l'idea evoluzionistica della storia dell'arte di Senocrate di Atene, la quale segue un serrato progredire dell'arte verso il naturalismo, e dall'altra invece la visione retrospettiva di Apollodoro che fondò il neoclassicismo attico. Quest'ultimo ebbe il sopravvento nel periodo del tardo ellenismo ed inoltre influenzò in gran parte anche l'arte romana sia nell'età tardo repubblicana sia in quella augustea. Nellopera di Plinio queste due tendenze non soltanto convivono, ma addirittura si sovrappongono: la storia dell'arte dagli inizi fino al III secolo a.C. viene scritta secondo l'interpretazione di Senocrate, mentre per il periodo successivo sono citati gli artisti di tendenza neoattica concordemente alla visione di Apollodoro.

Per l'arte romana a lui contemporanea invece Plinio non ha alcuna parola di lode. Nel suo tempo l'avidità per il denaro e la ricerca sfrenata del lusso hanno inflitto un colpo mortale al nobile operare dell'arte. Lopera artistica infatti non viene più considerata per i suoi valori intrinseci, tecnici ed ideali, ma si attribuisce valore soltanto al pregio dei materiali con cui viene realizzata. Questo modo di valutare la qualità delle espressioni artistiche le svuotò di contenuto e ne causò la decadenza finale, dalla quale Plinio non vede alcun ritorno. 


\section{Appendix: Index auctorum citato da Plinio il Vecchio nel primo libro della Storia naturale}

Fonti per il XXXIII libro:

EX AUCTORIBUS Domitiano Caesare. Iunio Gracchano. L. Pisone. M. Varrone. Corvino. Attico Pomponio. Calvo Licinio. Cornelio Nepote. Muciano. Boccho. Fetiale. Fenestella. Valerio Maximo. Iulio Basso qui de medicina Graece scripsit. Sextio Nigro qui item.

EXTERNIS Theophrasto. Democrito. Iuba. Timaeo historico. Qui de medicina metallica scripserunt: Heraclide. Andrea. Diagora. Botrye. Archedemo. Dionysio. Aristogene. Democle. Mneside. Attalo medico. Xenocrate item. Theomnesto. Nymphodoro. Iolla. Apollodoro. Pasitele qui mirabilia opera scripsit. Antigono qui de toreutice scripsit. Menaechmo qui item.

Fonti riguardanti il XXXIV libro:

EX AUCTORIBUS L. Pisone. Antiate. Verrio. M. Varrone. Cornelio Nepote. Messala Rufo. Marso poeta. Boccho. Iulio Basso qui de medicina Graece scripsit. Sextio Nigro qui item. Fabio Vestale.

EXTERNIS Democrito. Metrodoro Scepsio. Menaechmo qui de toreutice scripsit. Xenocrate qui item. Antigono qui item. Duride qui item. Heliodoro qui Atheniensium anathemata scripsit. Pasitele qui marabilia opera scripsit. Timaeo. Qui de medicina metallica scripserunt: Nymphodoro. Iolla. Apollodoro. Andrea. Heraclide. Diagora. Botrye. Archedemo. Dionysio. Aristogene. Democle. Mneside. Xenocrate Zenonis. Theomnesto.

XXXV libro:

EX AUCTORIBUS Messala oratore. Messala sene. Fenestella. Attico. M. Varrone. Verrio. Nepote Cornelio. Deculone. Muciano. Melisso. Vitruvio. Cassio Severo. Longulano. Fabio Vestale. 
EXTERNIS Qui de pictura scripserunt: Pasitele. Apelle. Melanthio. Asclepiodoro. Euphranore. Heliodoro qui anathemata Atheniensium scripsit. Metrodoro qui de architectonice scripsit. Democrito. Theophrasto. Apione grammatico. Qui de metallica medicina scripserunt: Nymphodoro. Iolla. Apollodoro. Andrea. Heraclide. Diagora. Botrye. Arcehedmo. Dionysio. Aristogene. Democle. Mneside. Xenocrate Zenonis. Theomnesto.

XXXVI libro:

EX AUCTORIBUS M. Varrone. C. Galba. Cincio. Muciano. Nepote Cornelio. L. Pisone. Q. Tuberone. Fabio Vestale. Annio Fetiale. Fabiano. Seneca. Catone censorio. Vitruvio.

EXTERNIS Theophrasto. Pasitele. Iuba rege. Nicandro. Sotaco. Sudine. Alexandro polyhistore. Apione Plistonice. Duride. Herodoto. Euhemero. Aristagora. Dionysio. Artemidoro. Butorida. Antisthene. Demetrio. Demotele. Lycea.

XXXVII libro:

EX AUCTORIBUS M. Varrone. actis triumphorum. Maecenate. Iaccho. Cornelio Boccho.

EXTERNIS Iuba rege. Xenocrate Zenonis. Sudine. Aeschylo. Philoxeno. Euripide. Nicandro. Satyro. Theophrasto. Charete. Philemone. Demostrato. Zenothemi. Metrodoro. Sotaco. Pythea. Timaeo Siculo. Nicia. Theochresto. Asaruba. Mnasea. Theomene. Ctesia. Mithridate. Sophocle. Archelao rege. Callistrato. Democrito. Ismenia. Olympico. Alexandro polyhistore. Apione. Oro. Zoroastre. Zachalia. 


\section{NOTA BIBLIOGRAFICA. I CORPORA DELLE FONTI ANTICHE E PLINIO IL VECCHIO}

Le raccolte (corpora) delle fonti antiche che seguono il metodo della cronologia degli artisti e delle loro opere:

Brunn, H., Geschichte der griechischen Künstler, I-II, Braunschweig, 1853 (I), Stuttgart, 1859 (II).

Overbeck, J., Die antiken Schriftquellen zur Geschichte der bildenden Künste bei den Griechen, Leipzig, 1868 (la recente edizione Hildesheim, 1959).

Reinach, A., Textes Grecs et Latins relatifs à l'histoire de la peinture ancienne, Paris, 1921 (la recente edizione Paris, 1985: introduzione e note di Rouveret, A.).

Pollitt, J. J., The Art of Ancient Greece. Sources and Documents, Cambridge University Press, 1990 (la prima edizione 1965).

Pollitt, J. J., The Art of Rome, c. 753 B.C. - A.D. 337. Sources and Documents, Cambridge University Press, 1983 (la prima edizione 1966).

Stuart Jones, H., Select Passages from Ancient Writers Illustrative of the History of Greek Sculpture, Chicago, 1966.

Le raccolte (corpora) delle fonti antiche che seguono vari criteri di raccolta:

Becatti, G., Arte e gusto negli scrittori latini, Firenze, 1951.

Pollitt, J. J., The Ancient View of Greek Art. Criticism, History and Termino$\log y$, New Haven, London, 1974.

Patera, B., La letteratura sull'arte nell'antichità, Palermo, 1975.

Hebert, B. D., Schriftquellen zur hellenistischen Kunst. Plastik, Melerei und Kunsthandwerk der Grichen vom vierten bis zum zweiten Jahrhundert, Graz, 1989 (»Grazer Beiträge«, Suppl. Bd. 4, 1989).

Gualandi, M. L., Lantichità classica, Roma, 2001 (Le fonti per la storia dell'arte, 1). 


\section{Edizioni critiche per i soli libri storico-artistici della Storia naturale:}

Plinio il Vecchio, Storia delle arti figurative (testo critico, traduzione e commento di Ferri, S.), Roma, 1946 (la recente edizione Milano, 2000: introduzione di Harari, M.).

Gaio Plinio Secondo, Storia naturale, V. Mineralogia e storia dellarte. Libri 33-37 (traduzione e note di Corso, A., Mugellesi, R., Rosati, G.), Torino, 1988.

Pline l'Ancien, Histoire Naturelle. Livre XXXIV (testo critico, traduzione e commento di Le Bonniec, H., Gallet de Santerre, H.) Paris, $1983^{2}$.

Pline l'Ancien, Histoire Naturelle. Livre XXXV (testo critico, traduzione e commento di Croisille, J.-M.) Paris, 1985.

Pline l'Ancien, Histoire Naturelle. Livre XXXVI (testo critico, traduzione e commento di André, J., Bloch, R., Rouveret, A.), Paris, 1981.

\section{Su Plinio come fonte letteraria per l'arte antica:}

Ferri, S., Note esegetiche ai giudizi d'arte di Plinio il Vecchio, Annali della Scuola Superiore Normale di Pisa s. 2, 11 (1942), pp. 69-116.

Bieber, M., Pliny and the Graeco-Roman Art, in: Hommages à Joseph Bidez et à Franz Cumont, Bruxelles, 1949, pp. 39-42.

Plinio il Vecchio sotto il profilo storico e letterario (Atti del Convegno di Como, 5-7 ottobre 1979. Atti della Tavola Rotonda, Bologna 16 dicembre 1979), Como, 1982.

Plinio, i suoi luoghi, il suo tempo (Atti delle Conferenze), Como, 1984.

Isager, J., Pliny on Art and Society. The Elder Pliniy's Chapters on the History of Art, London, New York, 1991.

Rouveret, B., Artistes, collectionneurs et antiquaires. L'histoire de l'art dans l'encyclopédie plinienne, in: Historie de l'histoire de l'art, I. De l'Antiquité au XVIIIe siècle, Paris, 1995, pp. 49-64. 
Le fonti letterarie greche e romane per la storia dell'arte antica di Plinio:

Kalkmann, A., Die Quellen der Kunstgeschichte des Plinius, Berlin, 1898.

Moreno, P., Fonti letterarie per la storia dell'arte antica, Colloqui del Sodalizio s. 2, 4 (1973-1974), pp. 33-54.

Settis, S., La trattatistica delle arti figurative, in: Lo spazio letterario della Grecia antica, I, 2. L'ellenismo, Roma, 1993, pp. 469-498.

\section{Duride di Samo}

Moreno, P., s. v. Duride di Samo, in Enciclopedia dellarte antica classica e orientale, 3, Roma, 1960, col. 198.

Wegner, M., Duris. Ein kunstlermonographischer Versuch, Münster, 1968.

\section{Senocrate di Atene}

Schweitzer, B., Xenokrates von Athen. Beiträge zur Geschichte der antiken Kunstforschung und Kunstanschauung, Halle, 1932.

Moreno, P., s. v. Xenokrates, in: Enciclopedia dell'arte antica classica e orientale, 7, Roma, 1966, col. 1234.

\section{Antigono di Caristo}

von Wilamowitz-Moellendorf, U., Antigonos von Karystos, Berlin, 1881.

Dorandi, T., Prolegomeni per una edizione di frammenti di Antigono de Caristo II, Museum Helveticum 51 (1994), pp. 5-29.

\section{Pasitele}

Borda, M., La Scuola di Pasiteles, Bari,1953. 


\section{PLINY THE ELDER AND HIS HISTORY OF ANCIENT ART}

Keywords: Pliny the Elder, Naturalis Historia, Duris of Samos, Xenocrates of Athens, Antigonus of Carystus, Appolodorus of Athens, Pasiteles, Marcus Terentius Varro

\section{Abstract}

The literature on ancient art that appeared in the 6th century BC alongside the first art treatises, and later expanded its scope and diversity, has been almost completely lost. Only three works have been fully preserved, and even these only from the Roman period: Vitruvius' treatise De Architectura, Pliny the Elder's encyclopedia Naturalis Historia, which includes an excursus on ancient art in individual chapters, and Pausanias' Description of Greece intended for Roman educated travelers. In addition to these three works, two other types of texts are classified as literary sources of art history: the essays that belong to the genre of ekphrasis (rhetorically perfected descriptions of artistic objects, real or imaginary), and the corpus of excerpts by selected authors of Antiquity that generally wrote about other themes, but in places also talked about ancient art.

When Pliny the Elder composed his chapters on ancient art forms he used original works of art or Greek and Roman art history literature. When he was writing the Naturalis Historia he used information from numerous authors that he cited in the first book and also some parts of the text. The Roman encyclopedist drew his knowledge of technical aspects of art primarily from Greek treatises published between the 6th and 4th centuries BC by numerous artists; among them, the sculptor Polykleitus of Argos and the painter Pamphilus of Amphipolis are worthy of mention.

Art history literature that coincides at least in part with the modern concept of art history had its beginnings in the early Hellenistic period. Pliny based his work on three authors from this period: Duris of Samos, Xenocrates of Athens, and Antigonus of Carystus. Duris of Samos (close of the 4th century BC) designed his two works - which have the generic titles On Painters and On Sculptors - as a series of biographies, in which he 
highlighted the apex of every artist's life, coinciding with the creation of his most important work. In his essays, Xenocrates of Athens (second quarter of the 3rd century BC) presented the history of ancient art as a continuous evolutionary line in which great artists played the role of the first inventors. Thus art followed the development towards naturalism; that is, perfection personified, in Xenocrates' opinion, by Lysippos among the sculptors, and Apelles among the painters. Antigonus of Carystus (last quarter of the 3rd century BC) enriched the work of his two predecessors with his own epigraphic studies.

In the middle and late Hellenistic period, Xenocrates' developmental view of the world was a generally accepted idea. Individual authors differed only by the period in which they placed the apex of development. The most influential among them, Appolodorus of Athens (2nd century BC), put the Athenian artists of the Age of Pericles to the forefront; especially Phidias. Appolodorus' nostalgic and retrospective vision (in contrast to Xenocrates, who recognized the masters of Sicyon school, to which he also belonged, to be the pinnacle of art history development) coincided with the renewal of Attic art around $150 \mathrm{BC}$, known also as the Classicism of Athens or neoAtticism.

In the late Hellenistic period, various compendiums were popular (mainly periegeses and lists of artists), among which Pasiteles' (first half of the 1 st century BC) essay entitled Mirabilia is the most interesting.

Although Pliny the Elder based his history of ancient art on all the authors mentioned above, the writings of classical philologists and archeologists often imply that he might not have always used the art history literature mentioned as primary sources, but rather cited a great deal of information from the works of his predecessor, the famous Roman encyclopedist Marcus Terentius Varro (beginning of the 1st century BC) 